\title{
On the Hausdorff distance between the shifted Heaviside step function and the transmuted Stannard growth function
}

\author{
Anton Iliev ${ }^{* \dagger}$, Nikolay Kyurkchiev ${ }^{\dagger}$, Svetoslav Markov ${ }^{\dagger}$ \\ ${ }^{*}$ Faculty of Mathematics and Informatics \\ Paisii Hilendarski University of Plovdiv, Bulgaria \\ aii@uni-plovdiv.bg \\ ${ }^{\dagger}$ Institute of Mathematics and Informatics \\ Bulgarian Academy of Sciences, Sofia, Bulgaria \\ nkyurk@math.bas.bg, smarkov@bio.bas.bg
}

Received: 4 July 2016, accepted: 4 September 2016, published: 14 September 2016

Dedicated to Professor Roumen Anguelov on the occasion of his 60th anniversary

Abstract-In this paper we study the one-sided Hausdorff distance between the shifted Heaviside step-function and the transmuted Stannard growth function. Precise upper and lower bounds for the Hausdorff distance have been obtained. We present a software module (intellectual property) within the programming environment CAS Mathematica for the analysis of the growth curves. Numerical examples, illustrating our results are given, too.

Keywords-Transmuted Stannard growth function; Heaviside step function; Hausdorff distance; Upper and lower bounds.

\section{INTRODUCTION AND PRELIMINARIES}

The Stannard function finds numerous applications in many scientific fields, including population dynamics, bacterial growth, population ecology, plant biology, chemistry, agriculture, demography, financial mathematics, statistics and fuzzy set theory [1]-[5].
Definition 1. For $\gamma \in \mathbb{R}$ define the shifted Heaviside step function as [12]:

$$
h_{\gamma}(t)=\left\{\begin{array}{cc}
0, & \text { if } t<0 \\
{[0,1],} & \text { if } t=\gamma \\
1, & \text { if } t>\gamma
\end{array}\right.
$$

Definition 2. Define the shifted Stannard growth function $S(t)$ as [1]-[5]:

$$
S(t)=\frac{1}{\left(1+e^{\frac{-(\beta+k(t-\gamma))}{m}}\right)^{m}},
$$

where $\beta, k$ and $m \in \mathbb{R}$ are the growth parameters. We note that the slope of (2) at $t=\gamma$ is equal to:

$$
\frac{k e^{-\frac{\beta}{m}}}{\left(1+e^{-\frac{\beta}{m}}\right)^{m+1}} \text {. }
$$

Definition 3. A random variable $T$ is said to have a transmuted distribution if its cumulative distribution function $(c d f)$ is given by [6], [7]:

$$
G_{1}(t)=(1+\lambda) F_{1}(t)-\lambda F_{1}^{2}(t), \quad|\lambda| \leq 1,
$$

where $F_{1}(t)$ is the $c d f$ of the base distribution. 
A. Iliev et al., On the Hausdorff distance between the shifted Heaviside ...

" $\lambda$ - transmuting" of $(c d f)$ is a familiar technique from the field of probability distributions with application to insurance mathematics.

Definition 4. The Hausdorff distance $\rho(f, g)$ between two interval functions $f, g$ on $\Omega \subseteq \mathbb{R}$, is the distance between their completed graphs $F(f)$ and $F(g)$ considered as closed subsets of $\Omega \times \mathbb{R}$ [8], [9], [12].

More precisely, we have

$$
\begin{gathered}
\rho(f, g)=\max \left\{\sup _{A \in F(f)} \inf _{B \in F(g)}\|A-B\|,\right. \\
\left.\sup _{B \in F(g)} \inf _{A \in F(f)}\|A-B\|\right\},
\end{gathered}
$$

wherein $\|$.$\| is any norm in \mathbb{R}^{2}$, e. g. the maximum norm $\|(t, x)\|=\max \{|t|,|x|\}$; hence the distance between the points $A=\left(t_{A}, x_{A}\right), B=\left(t_{B}, x_{B}\right)$ in $\mathbb{R}^{2}$ is $\|A-B\|=\max \left(\left|t_{A}-t_{B}\right|,\left|x_{A}-x_{B}\right|\right)$.

Sigmoidal growth curves typically have three parts (phases, time intervals): lag, log and stationary parts. It is a challenging question to characterize mathematically these phases. The lag time (interval) is practically important in many medical and biotechnological applications as this time is responsible for the acceleration or inhibition of the process and the possibility of controlling the lag time depends on the understanding of the hidden mechanisms of the corresponding process [10], [11].

Usually the lag time is defined by means of the uniform distance between the sigmoidal function and the induced cut function. We propose a new definition for the lag time by means of the Hausdorff distance between the sigmoidal function and the induced step function.

In this work we prove estimates for the onesided Hausdorff approximation of the shifted Heaviside step-function by transmuted Stannard growth function.

Let us point out that the Hausdorff distance is a natural measuring criteria for the approximation of bounded discontinuous functions [12], [13].

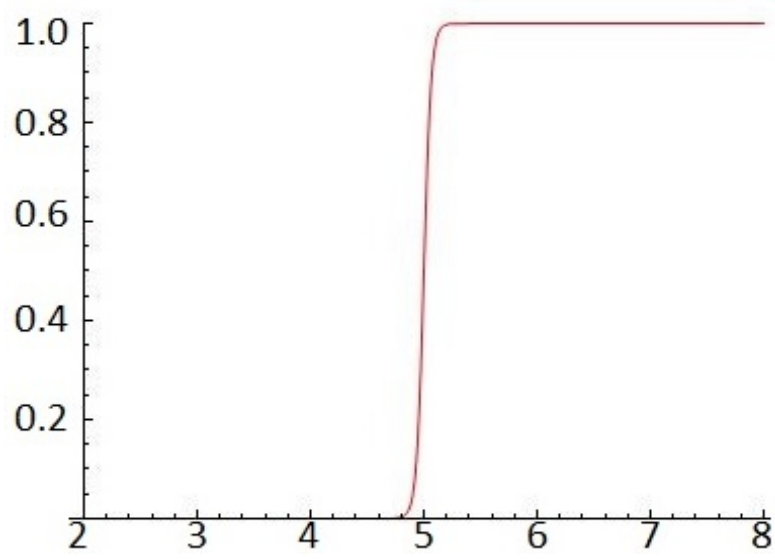

Fig. 1. Approximation of the shifted Heaviside step function by transmuted Stannard growth function for the following data: $k=16, m=0.52, \beta=0.01, t_{r}=5$; Hausdorff distance $d=0.0801797$.

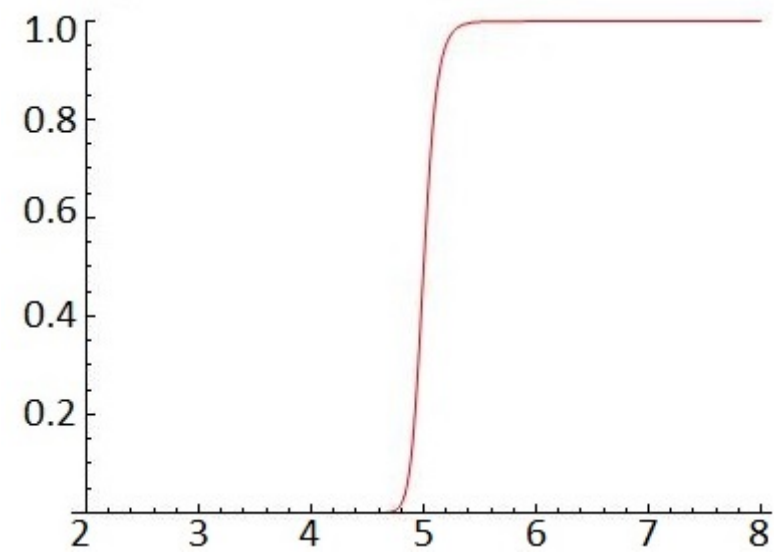

Fig. 2. Approximation of the shifted Heaviside step function by transmuted Stannard growth function for the following data: $k=26, m=2.1, \beta=1, t_{r}=5$; Hausdorff distance $d=0.112237$.

\section{MAin Results}

For $\gamma, \beta, m \in \mathbb{R}$ consider the following transmuted Stannard function

$$
\begin{aligned}
& S^{*}(t)=\frac{1+\lambda}{\left(1+e^{\frac{-(\beta+k(t-\gamma))}{m}}\right)^{m}}- \\
& \frac{\lambda}{\left(1+e^{\frac{-(\beta+k(t-\gamma))}{m}}\right)^{2 m}}, \quad|\lambda| \leq 1 .
\end{aligned}
$$


A. Iliev et al., On the Hausdorff distance between the shifted Heaviside ...

Function $S^{*}(t)$ from (5) satisfies:

$$
S^{*}(\gamma)=\frac{1+\lambda}{\left(1+e^{-\frac{\beta}{m}}\right)^{m}}-\frac{\lambda}{\left(1+e^{-\frac{\beta}{m}}\right)^{2 m}}=\frac{1}{2}
$$

hence

$$
\lambda=\frac{0.5(1+z)^{2 m}-(1+z)^{m}}{(1+z)^{m}-1} ; \quad z=e^{-\frac{\beta}{m}} .
$$

We study the Hausdorff approximation $d$ of the Heaviside step function $h_{\gamma}(t)$ by the transmuted Stannard function (5)-(7) and look for an expression for the error of the best one-sided approximation.

Let

$$
\begin{aligned}
A & =(1+\lambda)\left(1+e^{-\frac{\beta}{m}}\right)^{-m}-\lambda\left(1+e^{-\frac{\beta}{m}}\right)^{-2 m} \\
B & =1-2 e^{-\frac{\beta}{m}}\left(1+e^{-\frac{\beta}{m}}\right)^{-1-2 m} k \lambda \\
& +e^{-\frac{\beta}{m}}\left(1+e^{-\frac{\beta}{m}}\right)^{-1-m} k(1+\lambda), \quad k \in \mathbb{R} .
\end{aligned}
$$

The following Theorem gives upper and lower bounds for $d$.

Theorem 2.1 For the Hausdorff distance $d$ between the function $h_{\gamma}(t)$ and the transmuted Stannard function (5)-(7) the following inequalities hold for $|\lambda| \leq 1$ and $B>4$ :

$$
d_{l}=\frac{A}{2 B}<d<A \frac{\ln (2 B)}{2 B}=d_{r} .
$$

Proof. We need to express $d$ in terms of $k, \beta$ and $m$. The Hausdorff distance $d$ satisfies the relation

$$
\begin{gathered}
F(d):= \\
S^{*}(\gamma-d)=\frac{1+\lambda}{\left(1+e^{-\frac{\beta-k d}{m}}\right)^{m}}- \\
\frac{\lambda}{\left(1+e^{-\frac{\beta-k d}{m}}\right)^{2 m}}-d=0 .
\end{gathered}
$$

Consider the function

$$
G(d)=A-B d .
$$

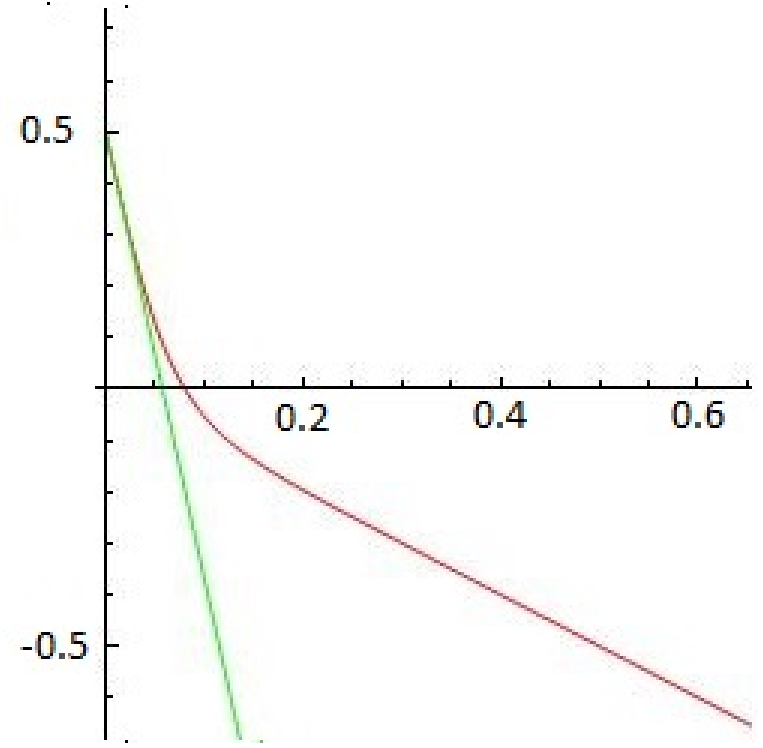

Fig. 3. The functions $F(d)$ and $G(d)$ for $k=16, m=0.52$, $\beta=0.01, t_{r}=5$.

By means of Taylor expansion we obtain

$$
G(d)-F(d)=O\left(d^{2}\right) .
$$

Hence $G(d)$ approximates $F(d)$ with $d \rightarrow 0$ as $O\left(d^{2}\right)$ (see Fig. 3). Further, for $|\lambda| \leq 1$ and $B>4$ we have

$$
\begin{aligned}
G\left(d_{l}\right) & =\frac{A}{2}>0, \\
G\left(d_{r}\right) & =A(1-0.5 \ln (2 B))<0 .
\end{aligned}
$$

This completes the proof of the theorem.

Some computational examples using relations (9) are presented in Table 1. The last column of Table 1 contains the values of $d$ computed by solving the nonlinear equation (10).

\begin{tabular}{|c|c|c|c|c|c|c|c|}
\hline$\beta$ & $k$ & $m$ & $\lambda$ & $\gamma$ & $d_{l}$ & $d_{r}$ & $d$ from(10) \\
\hline 1 & 26 & 2.1 & 0.594719 & 5 & 0.0480564 & 0.112559 & 0.112237 \\
0.1 & 24 & 1.3 & 0.300329 & 5 & 0.0407241 & 0.102127 & 0.10138 \\
0.01 & 16 & 0.52 & -0.957987 & 5 & 0.0287694 & 0.0821452 & 0.0801797 \\
0.63 & 21 & 0.875 & -0.997848 & 5 & 0.0317872 & 0.0875911 & 0.0793406 \\
0.1 & 28 & 0.7 & -0.63833 & 5 & 0.0228017 & 0.0704065 & 0.0692928 \\
\hline
\end{tabular}

TABLE I

BOUNDS FOR $d$ COMPUTED BY EQUATION (9) FOR VARIOUS $\beta, k, m$. 
A. Iliev et al., On the Hausdorff distance between the shifted Heaviside ...

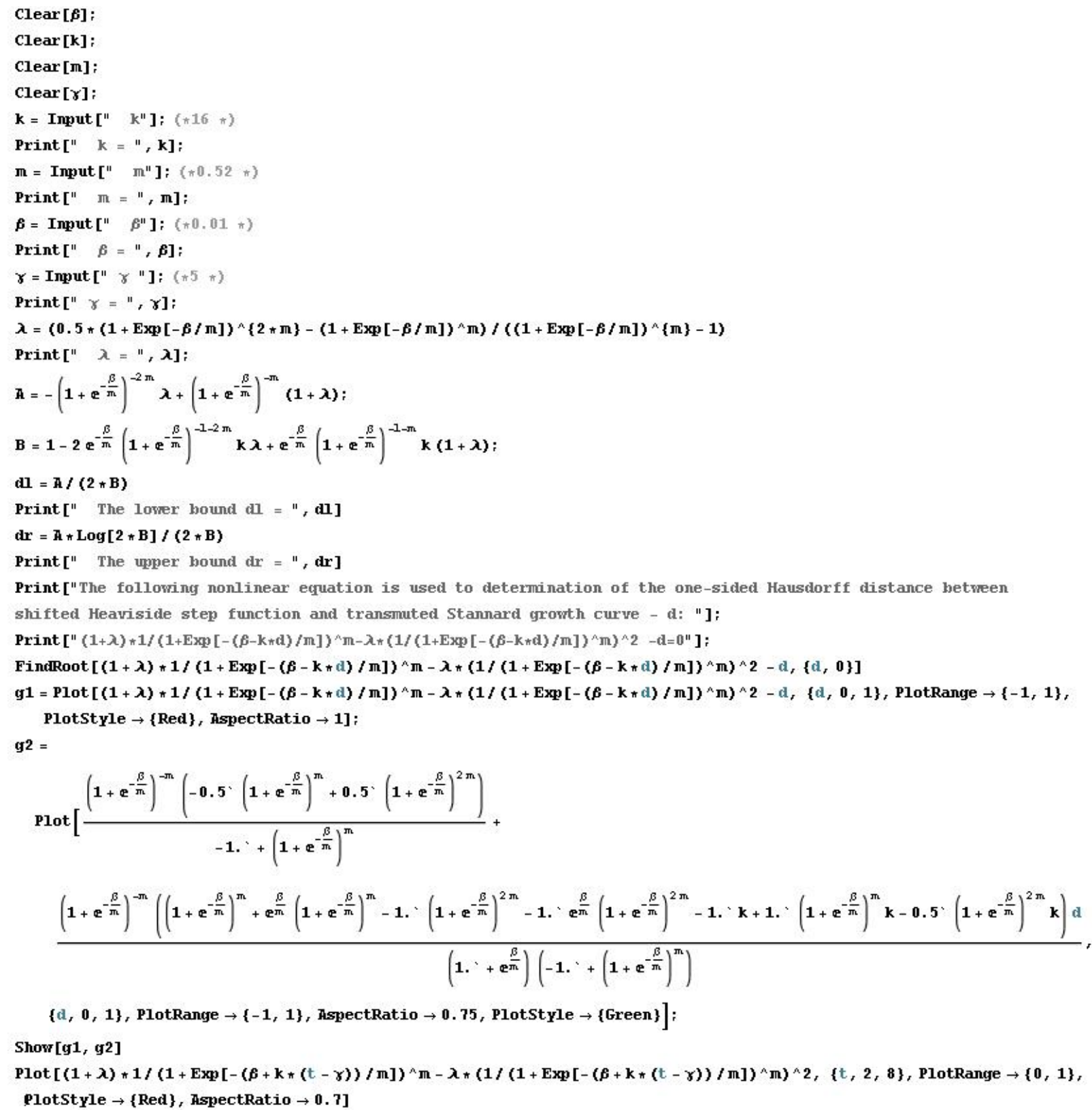

Fig. 4. A simple module implemented in CAS Mathematica for the computation and visualization of the Hausdorff distance between the Heaviside step function and the transmuted Stannard growth function. 
A. Iliev et al., On the Hausdorff distance between the shifted Heaviside ...

\section{CONCLUSION REMARKS}

New estimates for the Hausdorff distance between an interval Heviside step function and its best approximating Stannard function are obtained.

On Fig. 1 and Fig. 2 appropriate illustrations of some approximations of the shifted Heaviside step function by transmuted Stannard growth function are given.

We propose a software module within the programming environment CAS Mathematica for the analysis of the considered growth curves (see Fig. 4). The module offers the following possibilities:

i) generation of the shifted Stannard curve under user-defined values for $k, m, \beta$;

ii) automatic check of the condition $|\lambda| \leq 1$ that guarantees the existence of sigmoidality of the transmuted Stannard curve;

iii) software tools for animation and visualization.

The Hausdorff approximation of the interval step function by the logistic and other sigmoidal functions is discussed from various approximation, computational and modelling aspects in [14] $-[27]$.

\section{ACKNOWLEDGMENTS}

The authors would like to thank the anonymous reviewers for their helpful comments that contributed to improving the final version of the presented paper.

\section{REFERENCES}

[1] C. Stannard, A. Williams, P. Gibbs, Temperature/growth relationship for psychotropic foodspoilage bacteria, Food Microbiol. 2 (1985) 115-122.

[2] A. Khamis, Z. Ismail, Kh. Haron, A. Mohammed, Nonlinear growth models for modeling oil palm yield growth, Journal of Mathematics and Statistics 1(3) (2005) 225-233, doi:10.3844/jmssp.2005.225.232

[3] M. Zwietering, I. Jongenburger, F. Rombouts, K. Riet, Modeling of the Bacterial Growth Curve, Appl. and Environmental Microbiology 56(6) (1990) 1875-1881.

[4] M. Panik, Growth curve modeling: theory and applications, Wiley (2014), ISBN: 978-1-118-76404-6, doi:10.1002/9781118763971

[5] N. Kyurkchiev, A. Iliev, On some growth curve modeling: approximation theory and applications, Int. J. of Trends in Research and Development, 3(3) (2016) 466-471.
[6] W. Shaw, I. Buckley, The alchemy of probability distributions: beyond Gram-Charlier expansion and a skewkurtotic-normal distribution from rank transmutation map, Research report (2007).

[7] R. C. Gupta, O. Acman, S. Lvin, A sudy of log-logistic model in survival analysis, Biometrica Journal, 41(4) (1999) 431-443,

doi:10.1002/(SICI) 1521-4036 (199907)

$41: 4<431::$ AID-BIMJ431>3.0.CO;2-U

[8] F. Hausdorff, Set Theory (2 ed.), Chelsea Publ., New York (1962 [1957]) (Republished by AMS-Chelsea 2005), ISBN: 978-0-821-83835-8.

[9] B. Sendov, Hausdorff Approximations, Kluwer, Boston (1990) doi:10.1007/978-94-009-0673-0

[10] S. Shoffner, S. Schnell, Estimation of the lag time in a subsequent monomer addition model for fibril elongation, bioRxiv The preprint server for biology (2015) 1-8,

doi:10.1101/034900

[11] P. Arosio, T. P. J. Knowles, S. Linse, On the lag phase in amyloid fibril formation, Physical Chemistry Chemical Physics 17 (2015) 7606-7618, doi:10.1039/C4CP05563B

[12] R. Anguelov, S. Markov, Hausdorff Continuous Interval Functions and Approximations, In: M. Nehmeier et al. (Eds), Scientific Computing, Computer Arithmetic, and Validated Numerics, 16th International Symposium, Springer SCAN 2014, LNCS 9553 (2016) 3-13, doi:10.1007/978-3-319-31769-4

[13] N. Kyurkchiev, A. Andreev, Approximation and antenna and filter synthesis: Some moduli in programming environment Mathematica, LAP LAMBERT Academic Publishing, Saarbrucken (2014), ISBN 978-3659-53322-8.

[14] D. Costarelli, R. Spigler, Approximation results for neural network operators activated by sigmoidal functions, Neural Networks 44 (2013) 101-106, doi:10.1016/j.neunet.2013.03.015

[15] N. Kyurkchiev, On the Approximation of the step function by some cumulative distribution functions, Compt. rend. Acad. bulg. Sci. 68(12) (2015) 1475-1482.

[16] N. Kyurkchiev, S. Markov, On the Hausdorff distance between the Heaviside step function and Verhulst logistic function, J. Math. Chem. 54(1) (2016) 109-119, doi : 10.1007/S10910-015-0552-0

[17] N. Kyurkchiev, S. Markov, Sigmoidal functions: some computational and modelling aspects, Biomath Communications 1(2) (2014) 30-48, doi:10.11145/j.bmc.2015.03.081

[18] A. Iliev, N. Kyurkchiev, S. Markov, On the Approximation of the Cut and Step Functions by Logistic and Gompertz Functions, BIOMATH 4(2) (2015) 1510101, doi:10.11145/j.biomath.2015.10.101

[19] A. Iliev, N. Kyurkchiev, S. Markov, On the Approximation of the step function by some sigmoid functions, 
A. Iliev et al., On the Hausdorff distance between the shifted Heaviside ...

Mathematics and Computers in Simulation (2015),

doi:10.1016/j.matcom.2015.11.005

[20] N. Kyurkchiev, S. Markov, On the approximation of the generalized cut function of degree $p+1$ by smooth sigmoid functions, Serdica J. Computing 9(1) (2015) 101-112.

[21] N. Kyurkchiev, S. Markov, Sigmoid functions: Some Approximation and Modelling Aspects, LAP LAMBERT Academic Publishing, Saarbrucken (2015), ISBN 978-3-659-76045-7.

[22] V. Kyurkchiev, N. Kyurkchiev, On the Approximation of the Step function by Raised-Cosine and Laplace Cumulative Distribution Functions, European International Journal of Science and Technology 4(9) (2015) 75-84.

[23] N. Kyurkchiev, S. Markov, A. Iliev, A note on the
Schnute growth model, Int. J. of Engineering Research and Development 12(6) (2016) 47-54.

[24] N. Kyurkchiev, A. Iliev, A note on some growth curves arising from Box-Cox transformation, Int. J. of Engineering Works 3(6) (2016) 47-51.

[25] N. Kyurkchiev, A note on the new geometric representation for the parameters in the fibril elongation process, Compt. rend. Acad. bulg. Sci. 69(8) (2016) 963-972.

[26] D. Costarelli, G. Vinti, Pointwise and uniform approximation by multivariate neural network operators of the max-product type, Neural Networks (2016), doi:10.1016/j.neunet.2016.06.002

[27] Iliev, A., N. Kyurkchiev, S. Markov, Approximation of the cut function by Stannard and Richards sigmoid functions, IJPAM 109(1) 2016 119-128. 